\title{
Twisted Trace Formula for Hecke Correspondences
}

\author{
Salahoddin Shokranian* \\ Departamento de Matemática \\ Universidade de Brasília, Brazil \\ E-mail: sashemat.unb.br
}

By the twisted trace formula for the Hecke correspondences we understand the trace formulas for Hecke operators for a non-connected algebraic group acting on certain cohomology spaces. The traces of Hecke operators developed by Selberg in 1957 and the work of Arthur on the traces of Hecke operators of 1989 on $L^{2}$-cohomology are in some sense application of analysis and representation theory. On the other hand the work of Eichler in 1957 and of Goresky-MacPherson, Harder and Kottwitz from 1993 to present, reflects more geometric and topological applications. These may be seen as different solutions to the same problem, the calculation of the traces of Hecke operators. The present paper intends to be an introductory note about these operators and their applications.

Fourth International Winter Conference on Mathematical Methods in Physics 09 - 13 August 2004

Centro Brasileiro de Pesquisas Fisicas (CBPF/MCT), Rio de Janeiro, Brazil

${ }^{*}$ Speaker. 


\section{Introduction}

By the twisted trace formula for the Hecke correspondences we understand the trace formulas for Hecke operators for a non-connected algebraic group acting on certain cohomology spaces. The traces of Hecke operators developed by Selberg in 1957 and the work of Arthur on the traces of Hecke operators of 1989 on $L^{2}$-cohomology are in some sense application of analysis and representation theory. On the other hand the work of Eichler in 1957 and of Goresky-MacPherson, Harder and Kottwitz from 1993 to present, reflects more geometric and topological applications. These may be seen as different solution to the same problem, the calculation of the traces of Hecke operators. The present paper in which almost nothing is proved is an introduction to an unpublished long manuscript of mine [1], see also the recent paper of Goresky-MacPherson [2]. For almost all undefined notations we use those references and also [3].

In those works the main data are essentially:

- $G$ a connected reductive algebraic group defined over $\mathbf{Q}$,

- $\Gamma$ an arithmetic subgroup of $G(\mathbf{Z})$ of finite co-volume.

Under these assumptions a problem would be to extend the above theory to the non-connected groups. Thus the problem essentially is divided in two parts: to develop an analogue of the Selberg-Arthur trace formula for such groups, and to develop an analogue of the work of GoreskyMacPherson, Harder and Kottwitz.

\section{Basic set up}

The basic set up for our plan is the following:

- $G$ is a connected reductive group over $\mathbf{Q}$ with a $\mathbf{Z}$-structure, so that $G(\mathbf{Z})$ is defined and non-empty,

- $\varepsilon: G \rightarrow G$ an automorphism of finite order defined over $\mathbf{Q}$,

- $\theta$ a Cartan involution of $G(\mathbf{R})$ that commutes with $\varepsilon$. $K=K_{\varepsilon}$ the maximal compact subgroup of $G(\mathbf{R})$ associated to $\theta$. Then $\varepsilon(K)=K$,

- $\Gamma$ an arithmetic subgroup of $G(\mathbf{Z})$ such that $\varepsilon(\Gamma)=\Gamma$,

- The symmetric space $D=G(\mathbf{R}) / K$ and the locally symmetric variety $X_{\Gamma}=\Gamma \backslash D$,

- The non-connected group $G^{\prime}=G\langle\varepsilon\rangle$, the semidirect product of $G$ and the group $\langle\varepsilon\rangle$ generated by $\varepsilon$,

- A parabolic subgroup $P^{\prime}$ in $G^{\prime}$ is the normalizer $N_{G^{\prime}}^{\varepsilon}(P)$ of a parabolic subgroup $P$ of $G$ in $G^{\prime}$,

- We fix a minimal parabolic subgroup $P_{0}$ in $G$ and a Levi component $M_{0}$ of $P_{0}$. A parabolic subgroup $P$ of $G$ is called standard if $P_{0} \subset P$. It is called semi-standard if it contains $M_{0}$. The Levi subgroup of a standard parabolic subgroup is called a standard Levi subgroup of $G$, 
- A Levi subgroup in $G^{\prime}$ is the normalizer in $G^{\prime}$ of some standard Levi subgroup of $G$.

Lemma 1. If $P$ is an $\varepsilon$-stable parabolic subgroup of $G$ (i.e., $\varepsilon(P)=P$ ), then the parabolic subgroup $P^{\prime}=N_{G^{\prime}}^{\varepsilon}(P)$ is of the form $P\langle\varepsilon\rangle$.

Lemma 2. (a) If $P$ is an $\varepsilon$-stable parabolic subgroup of $G$, then $P^{\prime}=P\langle\varepsilon\rangle$ is a parabolic subgroup of $G^{\prime}$.

(b) If $P^{\prime}=P\langle\varepsilon\rangle$ is a parabolic subgroup in $G^{\prime}$, then $M^{\prime}=M\langle\varepsilon\rangle$ is a Levi component of $P^{\prime}$, provided $M$ is a Levi component of $P$ and $\varepsilon(M)=M$.

\section{The $\varepsilon$-action}

For any $g_{1} K \in G(\mathbf{R}) / K$ we define

$$
\varepsilon\left(g_{1} K\right)=\varepsilon\left(g_{1}\right) K
$$

and we define $\varepsilon$-action of $G(\mathbf{R})$ on $G(\mathbf{R}) / K$ by

$$
g \circ g_{1} K=g \varepsilon\left(g_{1}\right) K
$$

Note that this is not an action in the sense of $G$-sets. This is a (continuous) map from $G(\mathbf{R}) \times$ $G(\mathbf{R}) / K$ onto $G(\mathbf{R}) / K$.

Example 1. Let $G=S L(2), \theta(g)={ }^{t} g^{-1}$. Also let $\varepsilon=\theta$. Then $K=K_{\theta}=S O(2, \mathbf{R})$ and $G(\mathbf{R}) / K \simeq \mathbf{H}=\{z \in \mathbf{C} \mid \operatorname{Im}(z)>0\}$. The association of $g K$ to $z \in \mathbf{H}$ is as follows

$$
z=x+i y \leftrightarrow g=\left[\begin{array}{cc}
\sqrt{y} & \frac{x}{\sqrt{y}} \\
0 & \frac{1}{\sqrt{y}}
\end{array}\right] K .
$$

Also recall that $z=x+i y=g K \cdot i$ in the right hand side is ordinary action. Then the $\varepsilon$ - action on $g K$ can be written as

$$
\varepsilon(g) K=\left[\begin{array}{rr}
\frac{1}{\sqrt{y}} & 0 \\
-\frac{x}{\sqrt{y}} & \sqrt{y}
\end{array}\right] K .
$$

We call $\varepsilon(g) K, \varepsilon(z)$. We want to locate the position of $\varepsilon(z)$ in $\mathbf{H}$. This point is $w=\varepsilon(g) \cdot i$. Hence

$$
w=\left[\begin{array}{rr}
\frac{1}{\sqrt{y}} & 0 \\
-\frac{x}{\sqrt{y}} & \sqrt{y}
\end{array}\right] K \cdot i=\frac{i y-x}{x^{2}+y^{2}} \in \mathbf{H} .
$$

Hence $\varepsilon(z)=-\frac{1}{z}$.

\section{Hecke correspondences}

We let $\Gamma \subset G(\mathbf{Z})$ be an $\varepsilon$-stable subgroup. Let $\tilde{\Gamma}$ be the commensurator of $\Gamma$ in $G(\mathbf{Q})$, i.e

$$
\tilde{\Gamma}=\left\{g \in G(\mathbf{Q}) \mid \Gamma \sim g^{-1} \Gamma g\right\}
$$


where $\sim$ stands for commensurability among subgroups. Let $g$ be an element of $\tilde{\Gamma}$ such that $\Gamma^{\prime}:=$ $\Gamma \cap g^{-1} \Gamma g$ be $\varepsilon$-stable. Definition 1. A twisted Hecke correspondence is a "correspondence" $T_{\varepsilon}(g)$ defined by two maps $c_{1}$ and $c_{2}$ such that

$$
c_{1}\left(\Gamma^{\prime} y\right)=\Gamma \varepsilon(y), c_{2}\left(\Gamma^{\prime} y\right)=\Gamma g y,
$$

here $\varepsilon(y)=\varepsilon(h) K$, with $y=h K$, and

$$
c_{i}: \Gamma^{\prime} \backslash D \rightarrow \Gamma \backslash D, \quad i=1,2 .
$$

The role of the Hecke correspondences in the topological aspect of the trace formula that may also be called the Topological Trace Formula is the study of the Lefschetz theory for the fixed points of the Hecke correspondences. This is the same situation that happens in the case of the Hecke correspondences for the non-connected groups. For this we need to determine the fixed points of the twisted Hecke correspondences. This set by definition equals the set of all points in $D$ such that their images coincide. In another words we have

$$
\operatorname{Fix}\left(T_{\varepsilon}(g)\right):=\{y \in D \mid \Gamma \varepsilon(y)=\Gamma g y\} .
$$

Lemma 3. $y \in \Gamma \backslash D$ is a fixed point for $T_{\varepsilon}(g)$ if and only if $g^{-1} \gamma$ is $\varepsilon$-conjugate to an element of $K$ for certain $\gamma \in \Gamma$.

We recall that two elements $u, v \in G(\mathbf{R})$ are $\varepsilon$-conjugate if there is an element $h \in G(\mathbf{R})$ such that $v=h^{-1} u \varepsilon(h)$.

\section{Twisted centralizer and the fixed points}

We can relate twisted Hecke correspondences to harmonic analysis by using adelic language. Let $\mathbf{A}$ be the ring of adeles of $\mathbf{Q}$ and $\mathbf{A}_{f}$ the subring of finite adeles. For each standard parabolic subgroup $P$ of $G$ we denote by $K_{\theta, M}$ the intersection $K_{\theta, M}: K_{\infty} \cap M(\mathbf{R})$, where $K_{\theta}$ is the maximal compact subgroup of the set of fixed points under the action by the Cartan involution $\theta$. Let $A_{M}$ be a maximal Q-split torus in $M$. Let

$$
X_{P}\left(K_{f}\right)=M(\mathbf{Q}) \backslash\left[\left(N\left(\mathbf{A}_{f}\right) \backslash G\left(\mathbf{A}_{f}\right) / K_{f}\right) \times D_{M}\right],
$$

with

$$
D_{M}=M(\mathbf{R}) /\left(K_{\theta, M} \cdot A_{M}(\mathbf{R})^{\circ}\right),
$$

where $K_{f}$ is an small open subgroup in $G\left(\mathbf{A}_{f}\right)$ whose associated arithmetic subgroup is neat. Now we see that when $P=G$ then

$$
X\left(K_{f}\right)=X_{G}\left(K_{f}\right)=G(\mathbf{Q}) \backslash\left[\left(G\left(\mathbf{A}_{f}\right) / K_{f}\right) \times D\right],
$$

with $G=G(\mathbf{R}) / K_{\theta}$.

Definition 2. The spaces $X_{P}\left(K_{f}\right)$ are called the Borel-Serre stratum of $X\left(K_{f}\right)$. The union

$$
X^{r}\left(K_{f}\right)=X\left(K_{f}\right) \cup_{P \neq G}\left(X_{P}\left(K_{f}\right)\right)
$$


is the reductive Borel-Serre compactification of $X\left(K_{f}\right)$.

One has to find out the structure of the fixed point set of the twisted Hecke correspondence on the Borel-Serre stratum. The following is useful.

Lemma 4. Let $Y_{\varepsilon}$ be the set of all fixed points of the $\varepsilon$-action of $\gamma$ on $D_{M}$. Let

$$
M_{\gamma, \varepsilon}(\mathbf{Q})=\left\{u \in M(\mathbf{Q}) \mid u^{-1} \gamma \varepsilon(u)=\gamma\right\},
$$

be the twisted centralizer of $\gamma$. There is a subset $Y^{\varepsilon} \subset M\left(\mathbf{A}_{f}\right)$ so that the fixed points set of the twisted Hecke correspondence is given by

$$
\operatorname{Fix}(P, \varepsilon, \gamma)=M_{\gamma, \varepsilon}(\mathbf{Q}) \backslash\left(Y_{\varepsilon} \times Y^{\varepsilon}\right) \text {. }
$$

\section{Weighted cohomology}

Weighted cohomology generalizes the cohomology of arithmetic groups, the intersection cohomology and $L^{2}$-cohomology. It has both geometric applications and arithmetic properties. It is build up from hypercomplexes and a main ingredient is the weight profile. A weight profile is a function from the conjugacy classes of maximal rational parabolic subgroups of $G$ to the set of all half-integers

$$
\mathbf{Z}+\frac{1}{2}=\left\{\cdots,-\frac{3}{2},-\frac{1}{2}, \frac{1}{2}, \frac{3}{2}, \cdots\right\}
$$

\section{Euler-Poincaré characteristic}

In general an Euler-Poincaré characteristic of a cohomology theory with finite rank (dimension) is a finite alternating sum of the dimensions of the cohomology spaces, which are assumed to be finite of course.

The cohomology spaces of the weighted cohomology hypercomplex $W^{p} H\left(\Gamma, V_{\mu}\right)$ with weight profile $p$ and $i$-th cohomology $H$ with coefficients in a representation space $V_{\mu}$ gives rise to the following weighted Euler-Poincaré characteristic

$$
W^{p} \chi(\Gamma)=\sum_{i=0}^{\operatorname{dim} X}(-1)^{i} \operatorname{dim}\left(W^{p} H^{i}\left(X^{r}\left(K_{f}\right), V_{\mu}\right)\right)
$$

In general Euler-Poincaré characteristics have strong connection with number theory through their relations with the zeta functions and the rationality of such functions: the work of Gauus-Bonnet, Serre, Harder, and others.

\section{The Lefschetz formula}

To be able to get advantage of the known results on the trace of Hecke operators we have to relate Hecke operators to the Hecke correspondences. We can do so by rewriting the Hecke correspondences and Hecke operators in the adelic language, using their integral representations. The role of the twisted weighted orbital integrals is inevitable and the comparison of those integrals 
leads to the following result which essentially shows that the Lefschetz number for Hecke correspondences is the same as the trace formula for Hecke operators:

Theorem. The Lefschetz number for the alternating sum of the self-maps of twisted Hecke correspondences is given by an explicit formula that involves summation over Levi components, of a coefficient (explicitly calculable), time the twisted weighted orbital integrals. This formula may be obtained by the application of the Arthur trace formula for the calculation of the traces of Hecke operators for non-connected groups.

\section{References}

[1] S. Shokranian, Lefschetz Numbers on Weighted Cohomology for Twisted Groups, preprint 1999.

[2] M. Goreski and R. MacPherson, The Topological Trace Formula, J. Reine Angew. Math. 560 (2003) 77.

[3] S. Shokranian, The Selberg-Arthur Trace Formula, LNM 1503, Springer Verlag, Berlin 1992. 